\title{
THE GENERALIZED POLARIZATION TENSORS FOR RESOLVED IMAGING. PART I: SHAPE RECONSTRUCTION OF A CONDUCTIVITY INCLUSION
}

\author{
HABIB AMMARI, HYEONBAE KANG, MIKYOUNG LIM, AND HABIB ZRIBI
}

\begin{abstract}
With each $\mathcal{C}^{2}$-domain and material parameter, an infinite number of tensors, called the Generalized Polarization Tensors (GPTs), is associated. The GPTs contain significant information on the shape of the domain and its material parameter. They generalize the concept of Polarization Tensor (PT), which can be seen as the first-order GPT. It is known that given an arbitrary shape, one can find an equivalent ellipse or ellipsoid with the same PT. In this paper we consider the problem of recovering finer details of the shape of a given domain using higher-order polarization tensors. We design an optimization approach which solves the problem by minimizing a weighted discrepancy functional. In order to compute the shape derivative of this functional, we rigorously derive an asymptotic expansion of the perturbations of the GPTs that are due to a small deformation of the boundary of the domain. Our derivations are based on the theory of layer potentials. We perform some numerical experiments to demonstrate the validity and the limitations of the proposed method. The results clearly show that our approach is very promising in recovering fine shape details.
\end{abstract}

\section{INTRODUCTION}

With each shape of a domain, physical and geometric quantities, such as eigenvalues and capacity, are intrinsically associated. The notion of (generalized) polarization tensors (GPTs) is one of them [6]. The GPTs generalize the concepts of classic polarization tensors [25. The GPTs associated with a domain and a material parameter can be used to describe the perturbations of electric fields due to the presence of a conductivity inclusion. An electrical field present in a background homogeneous conductor is perturbed by the presence of the inclusions. Then the field perturbations can be represented by multipolar expansions which are expressed in terms of the GPTs. Indeed, the GPTs of the conductivity inclusion can be reconstructed from far-field measurements [6]. Consider the following conductivity transmission problem:

$$
\begin{cases}\nabla \cdot\left(\chi\left(\mathbb{R}^{2} \backslash \bar{D}\right)+k \chi(D)\right) \nabla u=0 & \text { in } \mathbb{R}^{2}, \\ u(x)-H(x)=O\left(|x|^{-1}\right) & \text { as }|x| \rightarrow \infty,\end{cases}
$$

Received by the editor August 18, 2010 and, in revised form, December 2, 2010.

2010 Mathematics Subject Classification. Primary 35R30, 49Q10, 49Q12.

Key words and phrases. Generalized polarization tensor, asymptotic expansions, shape recovery. 
where $\chi(D)$ denotes the characteristic function of the domain $D$ and $H$ is a given harmonic function in $\mathbb{R}^{2}$.

The coefficient $\chi\left(\mathbb{R}^{2} \backslash \bar{D}\right)+k \chi(D)$ represents the conductivity distribution. The inclusion $D$ has conductivity $k \neq 1$ while the background $\mathbb{R}^{2} \backslash \bar{D}$ has conductivity 1. The function $\nabla H$ is the background electric field and $\nabla u$ is the electric field in the presence of the inclusion $D$. Then the perturbation, $u-H$, is given by the multipolar expansion [6]:

$$
(u-H)(x)=\sum_{|\alpha|,|\beta|=1}^{+\infty} \frac{(-1)^{|\alpha|}}{\alpha ! \beta !} \partial^{\alpha} \Gamma(x) M_{\alpha \beta} \partial^{\beta} H\left(x_{0}\right) \quad \text { as }|x| \rightarrow+\infty,
$$

where $x_{0}$ is the center of mass of $D$ and $\Gamma$ is the fundamental solution to the Laplacian, i.e.,

$$
\Gamma(x)=\frac{1}{2 \pi} \ln |x| .
$$

Here $\alpha=\left(\alpha_{1}, \alpha_{2}\right)$ and $\beta=\left(\beta_{1}, \beta_{2}\right)$ are multi-indices and $|\alpha|=\alpha_{1}+\alpha_{2}$.

The quantity $M_{\alpha \beta}$ is called the generalized polarization tensor (GPT). Formula (1.2) shows that through the GPTs we have complete information about the far-field expansion of the perturbation $u-H$.

When $|\alpha|=|\beta|=1$, we denote $M_{\alpha \beta}=m_{i j}$ and call the matrix $M=\left(m_{i j}\right)_{i, j=1}^{2}$ the polarization tensor $(\mathrm{PT})$.

The concepts of PT and GPTs occur in several interesting contexts, in particular, in asymptotic models of dilute composites (see [22] and [10]) and in potential theory related to certain questions arising in hydrodynamics [25].

Another important use of these concepts is for imaging diametrically small inclusions from boundary measurements. In fact, the GPTs are the basic building blocks for the asymptotic expansions of the boundary voltage perturbations due to the presence of small conductivity inclusions inside a conductor [19, 3]. Based on this expansion, efficient algorithms to determine the location and some geometric features of the inclusions were proposed. We refer to [5, 6] and the references therein for recent developments of this theory.

According to [13] and [8, the PT associated with an unknown inclusion can be detected from boundary measurements. The detected PT in turn yields the "equivalent ellipse" of a single inclusion. In other words, in terms of the PT associated with an inclusion and a conductivity parameter (or a cluster of inclusions and a set of conductivity parameters) we are able to recover an equivalent ellipse with the same PT. On the other hand, it is proved in [4] that the full set of GPTs uniquely determines the inclusion (and its conductivity). In fact, from the set of all the GPTs we can recover the "Dirichlet-to-Neumann map" of the inclusion, and hence its shape and its conductivity, by making use of a uniqueness result due to Isakov 21. Therefore, it is natural to ask the question whether we can recover more shape details than the equivalent ellipse using a finite number of GPTs. The aim of this paper is to investigate this challenging question.

Recall that there is a canonical one-to-one correspondence between the class of PTs and the class of ellipses [13. That is why one can find easily the equivalent ellipse if one knows the PT. However, there is no (and it is unlikely to have one) known class of geometric shapes which has such a property for higher-order polarization tensors. In this paper, we propose an optimization approach to recover finer shape details using GPTs. 
Let $B$ be an unknown domain. Let $M_{\alpha \beta}(k, B)$ denote the GPT associated with $B$ and the conductivity $k$. It is worth emphasizing that the GPT also depends on the conductivity contrast $k$. Suppose that $M_{\alpha \beta}(k, B)$ are known for all $|\alpha|+|\beta| \leq K$ for some number $K$. Suppose also that the conductivity is known. Our recursive optimization procedure would be to minimize over $D$,

$$
J^{(n)}[D]:=\frac{1}{2} \sum_{|\alpha|+|\beta| \leq K} w_{|\alpha|+|\beta|}^{(n)}\left|\sum_{\alpha, \beta} a_{\alpha} b_{\beta} M_{\alpha \beta}(k, D)-\sum_{\alpha, \beta} a_{\alpha} b_{\beta} M_{\alpha \beta}(k, B)\right|^{2} .
$$

Here the coefficients $a_{\alpha}$ and $b_{\beta}$ are such that $H=\sum a_{\alpha} x^{\alpha}$ and $F=\sum b_{\beta} x^{\beta}$ are homogeneous harmonic polynomials and $w_{|\alpha|+|\beta|}^{(n)}$ are binary weights. We assign either 0 or 1 to $w_{|\alpha|+|\beta|}^{(n)}$ depending which GPTs are used at step $n$.

In step $n$ we use as an initial guess the result of step $n-1$. In the first step we get an equivalent ellipse with the same PT as well as the location of the inclusion. If there are multiple inclusions, we choose in the second step,

$$
w_{|\alpha|+|\beta|}^{(2)}=1 \quad \text { for } 3 \leq|\alpha|+|\beta| \leq K
$$

in order to have a better initial guess than an ellipse. In general, since we don't know whether the target is connected or multiple connected, we use the second choice of weights.

It is worth making a remark to motivate the use of linear combinations of the GPTs where the coefficients come from harmonic polynomials. Note that if $D$ is a ball, then the GPTs are the coefficients of the "Dirichlet-to-Neumann map" of $D$ in the basis of spherical harmonics. The reason that only the linear combinations corresponding to harmonic polynomials are useful is that they are the only ones corresponding to functions solving the background problem.

Our method is in the same spirit as the continuation method in frequency [14, 15, 11] which was designed to solve inverse scattering problems for the Helmholtz equation.

In order to minimize the weighted discrepancy functional given in (1.4), we need a shape derivative for the GPTs. It turns out that the shape derivative of $\sum_{\alpha, \beta} a_{\alpha} b_{\beta} M_{\alpha \beta}(k, D)$ has a simple form. This is the main reason of choosing as a discrepancy functional the difference between calculated and given harmonic sums of GPTs rather than the difference between individual GPTs. In order to calculate the shape derivative of our discrepancy functional, we derive an asymptotic expansion of the GPTs under small perturbations of the boundary of the inclusion $D$.

The derivation is rigorous and based on layer potential techniques in the same spirit as in [5, 6. We mention that related asymptotic formulas for boundary measurements, far-field data, and modal measurements have been obtained in a series of recent papers [9, 1, 2, 24].

We implement the proposed optimization procedure to recover both convex or non-convex shapes. The method of this paper is quite promising in the sense that the numerical results clearly exhibit that the shape moves toward the actual shape. They show not only the validity of the method but also that the equivalent ellipse is a good initial guess.

This paper is organized as follows. In Section 2, we review some basic facts on layer potentials which will be used to define the GPTs and to derive their 
shape derivatives. In Section 3 we review asymptotic formulas for perturbations in boundary integral operators due to small changes of the boundary. Section 4 is to derive a new asymptotic formula for the perturbations of the GPTs. In Section 5 we set up the optimization problem to recover shape details using a set of GPTs. In Section 6 we present results of numerical experiments and discuss the validity and the limitations of our method.

We emphasize that even though we only investigate the problem in two dimensions, the method of this paper is expected to work equally well in three dimensions.

\section{LAyer Potentials AND GPTs}

Throughout this paper we assume that the domains under consideration have $\mathcal{C}^{2}$-smooth boundaries. For a given bounded domain $D$ in $\mathbb{R}^{2}$, the single and double layer potentials of the density function $\phi \in L^{2}(\partial D)$ are defined by

$$
\begin{aligned}
\mathcal{S}_{D}[\phi](x) & :=\int_{\partial D} \Gamma(x-y) \phi(y) d \sigma(y), \quad x \in \mathbb{R}^{2}, \\
\mathcal{D}_{D}[\phi](x) & :=\int_{\partial D} \frac{\partial}{\partial \nu_{y}} \Gamma(x-y) \phi(y) d \sigma(y), \quad x \in \mathbb{R}^{2} \backslash \partial D,
\end{aligned}
$$

where $\nu_{y}$ is the outward unit normal to $\partial D$ at $y \in \partial D$ and $\Gamma$ is given by (1.3).

For a function $u$ defined on $\mathbb{R}^{2} \backslash \partial D$, we denote

$$
\left.\frac{\partial u}{\partial \nu}\right|_{ \pm}(x):=\lim _{t \rightarrow 0^{+}}\left\langle\nabla u\left(x \pm t \nu_{x}\right), \nu_{x}\right\rangle, \quad x \in \partial D,
$$

if the limits exist. The notation $\left.u\right|_{ \pm}$is understood likewise. The following are the well-known properties of the single and double layer potentials:

- Trace formula [20]:

$$
\begin{aligned}
\left.\frac{\partial \mathcal{S}_{D}[\phi]}{\partial \nu}\right|_{ \pm}(x) & =\left( \pm \frac{1}{2} I+\mathcal{K}_{D}^{*}\right)[\phi](x), \quad x \in \partial D, \\
\left.\mathcal{D}_{D}[\phi]\right|_{ \pm} & =\left(\mp \frac{1}{2} I+\mathcal{K}_{D}\right)[\phi](x), \quad x \in \partial D,
\end{aligned}
$$

where

$$
\mathcal{K}_{D}[\phi](x)=\frac{1}{2 \pi} \int_{\partial D} \frac{\langle y-x, \nu(y)\rangle}{|x-y|^{2}} \phi(y) d \sigma(y)
$$

and $\mathcal{K}_{D}^{*}$ is the $L^{2}$-adjoint of $\mathcal{K}_{D}$, i.e.,

$$
\mathcal{K}_{D}^{*}[\phi](x)=\frac{1}{2 \pi} \int_{\partial D} \frac{\langle x-y, \nu(x)\rangle}{|x-y|^{2}} \phi(y) d \sigma(y) .
$$

- For any real number $\lambda$ with $|\lambda|>1 / 2$ or $\lambda=-1 / 2,\left(\lambda I-\mathcal{K}_{D}^{*}\right)$ is invertible on $L^{2}(\partial D)$. If $|\lambda| \geq 1 / 2$, then $\left(\lambda I-\mathcal{K}_{D}^{*}\right)$ is invertible on $L_{0}^{2}(\partial D):=\{f \in$ $\left.L^{2}(\partial D): \int_{\partial D} f d \sigma=0\right\}$. See [20] and [26].

- If $\phi \in \mathcal{C}^{1, \alpha}(\partial D)$ for some $\alpha>0$, then $\mathcal{D}_{D} \phi$ is $\mathcal{C}^{1, \alpha}$ on $\bar{D}$ and $\mathbb{R}^{2} \backslash D$, and we have (see [16] for example)

$$
\left.\frac{\partial\left(\mathcal{D}_{D} \phi\right)}{\partial \nu}\right|_{-}=\left.\frac{\partial\left(\mathcal{D}_{D} \phi\right)}{\partial \nu}\right|_{+} \text {on } \partial D
$$


Let $D$ be a bounded domain in $\mathbb{R}^{2}$ and suppose that the conductivity of $D$ is $k$, $0<k \neq 1<+\infty$. Let $\lambda=(k+1) /(2(k-1))$. For a multi-index $\alpha=\left(\alpha_{1}, \alpha_{2}\right) \in \mathbb{N}^{2}$, define $\phi_{\alpha}$ by

$$
\phi_{\alpha}(y):=\left(\lambda I-\mathcal{K}_{D}^{*}\right)^{-1}\left[\nu_{x} \cdot \nabla x^{\alpha}\right](y), \quad y \in \partial D .
$$

Here and throughout this paper, we use the conventional notation: $x^{\alpha}=x_{1}^{\alpha_{1}} x_{2}^{\alpha_{2}}$. Then, the generalized polarization tensors $M_{\alpha \beta}$ for $\alpha, \beta \in \mathbb{N}^{2}$ are defined, equivalently to (1.2), by

$$
M_{\alpha \beta}(k, D):=\int_{\partial D} y^{\beta} \phi_{\alpha}(y) d \sigma(y)
$$

Key properties of positivity and symmetry of the GPTs are studied in $[6$, Chapter 4]. We shall emphasize that what is important is not the individual terms $M_{\alpha \beta}$ but their harmonic combinations. A harmonic combination of GPTs is $\sum_{\alpha, \beta} a_{\alpha} b_{\beta} M_{\alpha \beta}$ where $\sum_{\alpha} a_{\alpha} x^{\alpha}$ and $\sum_{\beta} b_{\beta} x^{\beta}$ are harmonic polynomials. We will call such $\left(a_{\alpha}\right)$ and $\left(b_{\beta}\right)$ harmonic coefficients. For example, the following symmetry property holds:

$$
\sum_{\alpha, \beta} a_{\alpha} b_{\beta} M_{\alpha \beta}(k, D)=\sum_{\alpha, \beta} a_{\alpha} b_{\beta} M_{\beta \alpha}(k, D)
$$

for any pair $\left(a_{\alpha}\right),\left(b_{\beta}\right)$ of harmonic coefficients.

Let us record the following uniqueness theorem.

Theorem 2.1. If all harmonic combinations of GPTs of two domains are the same, i.e.,

$$
\sum_{\alpha, \beta} a_{\alpha} b_{\beta} M_{\alpha \beta}\left(k_{1}, D_{1}\right)=\sum_{\alpha, \beta} a_{\alpha} b_{\beta} M_{\alpha \beta}\left(k_{2}, D_{2}\right)
$$

for all pairs $\left(a_{\alpha}\right),\left(b_{\beta}\right)$ of harmonic coefficients, then $D_{1}=D_{2}$ and $k_{1}=k_{2}$.

In [4], the uniqueness theorem was stated under the assumption that $M_{\alpha \beta}\left(k_{1}, D_{1}\right)$ $=M_{\alpha \beta}\left(k_{2}, D_{2}\right)$ for all $\alpha$ and $\beta$; but a quick glance of the proof there reveals that Theorem 2.1 is what was actually proved.

\section{ASYMPTOTIC EXPANSIONS OF BOUNDARY INTEGRAL OPERATORS}

Let $D$ be a bounded domain with $\mathcal{C}^{2}$-boundary and let, for $\epsilon$ small, $D_{\epsilon}$ be an $\epsilon$-perturbation of $D$, i.e., there is a function $h \in \mathcal{C}^{1}(\partial D)$ such that

$$
\partial D_{\epsilon}:=\{\tilde{x}=x+\epsilon h(x) \nu(x) \mid x \in \partial D\}
$$

where $\nu$ is the outward unit normal vector field on $\partial D$. Let $\Psi_{\epsilon}$ be the diffeomorphism from $\partial D$ to $\partial D_{\epsilon}$ given by

$$
\Psi_{\epsilon}(x)=x+\epsilon h(x) \nu(x) .
$$

In view of (2.3) and (2.4), we need to get an asymptotic expansion of the operator $\mathcal{K}_{D_{\epsilon}}^{*}$ in order to get that of $M_{\alpha \beta}\left(k, D_{\epsilon}\right)$. A complete asymptotic expansion of the boundary integral operator $\mathcal{K}_{D_{\epsilon}}^{*}$ on $L^{2}\left(\partial D_{\epsilon}\right)$ is derived in terms of $\epsilon$ in 9 , Theorem 2.1]. In particular, the first order approximation is as follows.

Lemma 3.1. For $\tilde{\phi} \in L^{2}\left(\partial D_{\epsilon}\right)$ let $\phi:=\tilde{\phi} \circ \Psi_{\epsilon}$. There exists a constant $C$ depending only on the $\mathcal{C}^{2}$-norm of $\partial D$ and $\|h\|_{\mathcal{C}^{1}}$ such that

$$
\left\|\left(\mathcal{K}_{D_{\epsilon}}^{*}[\tilde{\phi}]\right) \circ \Psi_{\epsilon}-\mathcal{K}_{D}^{*}[\phi]-\epsilon \mathcal{K}_{D}^{(1)}[\phi]\right\|_{L^{2}(\partial D)} \leq C \epsilon^{2}\|\phi\|_{L^{2}(\partial D)},
$$


with the operator $\mathcal{K}_{D}^{(1)}$ defined for any $\phi \in L^{2}(\partial D)$ by

$$
\mathcal{K}_{D}^{(1)}[\phi](x)=\text { p.v. } \int_{\partial D} \mathbb{k}_{1}(x, y) \phi(y) d \sigma(y) \quad x \in \partial D,
$$

where

$$
\begin{aligned}
\mathbb{k}_{1}(x, y)= & -2 \frac{\langle x-y, \nu(x)\rangle\langle x-y, h(x) \nu(x)-h(y) \nu(y)\rangle}{|x-y|^{4}} \\
& +\frac{\langle h(x) \nu(x)-h(y) \nu(y), \nu(x)\rangle}{|x-y|^{2}} \\
& -\frac{\left\langle x-y, \tau(x) h(x) \nu(x)+h^{\prime}(x) T(x)\right\rangle}{|x-y|^{2}} \\
& +\frac{\langle x-y, \nu(x)\rangle}{|x-y|^{2}}(h(x) \tau(x)-h(y) \tau(y)) .
\end{aligned}
$$

Here, $\tau(x)$ denotes the curvature of $\partial D$ at $x, T$ the unit tangential vector field on $\partial D$, p.v. the Cauchy principal value, and $h^{\prime}$ the derivative of $h$ on $\partial D$, i.e., $h^{\prime}=\frac{\partial h}{\partial T}$.

We shall emphasize that $\mathcal{K}_{D}^{(1)}$ is bounded on $L^{2}(\partial D)$. In fact, the first, second, and fourth kernels on the right-hand side of (3.4) are bounded since $\partial D$ is of class $\mathcal{C}^{2}$, while the third kernel defines a singular integral operator which is bounded on $L^{2}(\partial D)$ by the theorem of Coifman, McIntosh and Meyer [17.

Moreover, the following expansions of $\tilde{\nu}$ and $\tilde{\sigma}$ hold:

$$
\tilde{\nu}(\tilde{x})=\nu(x)-\epsilon h^{\prime}(x) T(x)+O\left(\epsilon^{2}\right)
$$

and

$$
d \tilde{\sigma}(\tilde{x})=d \sigma(x)-\epsilon \tau(x) h(x) d \sigma(x)+O\left(\epsilon^{2}\right) .
$$

Here, the remainder $O\left(\epsilon^{2}\right)$ is bounded by $C \epsilon^{2}$ for some $C$ which depends only on the $\mathcal{C}^{2}$-norm of $\partial D$ and $\|h\|_{\mathcal{C}^{1}(\partial D)}$.

The following lemma was also obtained in [9, Lemma 3.1].

Lemma 3.2. Let $\tilde{\phi}_{\epsilon}=\left(\lambda I-\mathcal{K}_{D_{\epsilon}}^{*}\right)^{-1}[\tilde{\nu} \cdot \nabla H], \phi_{\epsilon}=\tilde{\phi}_{\epsilon} \circ \Psi_{\epsilon}$, and $\phi=\left(\lambda I-\mathcal{K}_{D}^{*}\right)^{-1}[\nu$. $\nabla H]$. Then we have

$$
\left\|\phi_{\epsilon}-\phi-\epsilon \phi_{1}\right\|_{L^{2}(\partial D)} \leq C \epsilon^{2}\|\phi\|_{L^{2}(\partial D)}
$$

where $C$ is a constant depending only on the $\mathcal{C}^{2}$-norm of $\partial D$ and $\|h\|_{\mathcal{C}^{1}}$ and

$$
\phi_{1}=\left(\lambda I-\mathcal{K}_{D}^{*}\right)^{-1}\left[\mathcal{K}_{D}^{(1)}[\phi]+h\left\langle\left(\nabla^{2} H\right) \nu, \nu\right\rangle-h^{\prime} \frac{\partial H}{\partial T}\right] .
$$

We now rewrite the operator $\mathcal{K}_{D}^{(1)}$ in terms of more familiar operators. For $x, y \in \partial D(x \neq y)$, we have

$$
\begin{aligned}
\frac{\partial}{\partial T(x)} \Gamma(x-y) & =\frac{1}{2 \pi} \frac{\langle x-y, T(x)\rangle}{|x-y|^{2}} \\
\frac{\partial^{2}}{\partial T(x)^{2}} \Gamma(x-y) & =\frac{1}{2 \pi}\left[\frac{1}{|x-y|^{2}}+\frac{\langle x-y, \nu(x)\rangle \tau(x)}{|x-y|^{2}}-\frac{2(\langle x-y, T(x)\rangle)^{2}}{|x-y|^{4}}\right] \\
& =\frac{1}{2 \pi}\left[-\frac{1}{|x-y|^{2}}+\frac{\langle x-y, \nu(x)\rangle \tau(x)}{|x-y|^{2}}+\frac{2(\langle x-y, \nu(x)\rangle)^{2}}{|x-y|^{4}}\right],
\end{aligned}
$$


and

$$
\frac{\partial^{2}}{\partial \nu(x) \nu(y)} \Gamma(x-y)=\frac{1}{2 \pi}\left[-\frac{\langle\nu(x), \nu(y)\rangle}{|x-y|^{2}}+\frac{2\langle x-y, \nu(x)\rangle\langle x-y, \nu(y)\rangle}{|x-y|^{4}}\right] .
$$

It then follows that

$$
\begin{aligned}
- & \frac{\partial}{\partial T(x)}\left(h(x) \frac{\partial}{\partial T(x)}\right) \Gamma(x-y)+h(y) \frac{\partial^{2}}{\partial \nu(x) \nu(y)} \Gamma(x-y) \\
= & -2 \frac{\langle x-y, \nu(x)\rangle\langle x-y, h(x) \nu(x)-h(y) \nu(y)\rangle}{|x-y|^{4}}+\frac{\langle h(x) \nu(x)-h(y) \nu(y), \nu(x)\rangle}{|x-y|^{2}} \\
& -\frac{\left\langle x-y, \tau(x) h(x) \nu(x)+h^{\prime}(t) T(x)\right\rangle}{|x-y|^{2}} \\
= & \mathbb{k}_{1}(x, y)-\frac{\langle x-y, \nu(x)\rangle}{|x-y|^{2}}(h(x) \tau(x)-h(y) \tau(y)) .
\end{aligned}
$$

Define $H^{s}(\partial D), s=1,2$, to be the usual Sobolev spaces on $\partial D$. If $\phi \in H^{1}(\partial D)$, then $\mathcal{S}_{D}[\phi] \in H^{2}(\partial D)$ and $\frac{\partial}{\partial \nu} \mathcal{D}_{D}[h \phi] \in L^{2}(\partial D)$. Note that the left-hand side of the first identity is the integral kernel of the operator

$$
\phi \mapsto-\frac{\partial}{\partial T}\left(h \frac{\partial}{\partial T}\right) \mathcal{S}_{D}[\phi]+\frac{\partial}{\partial \nu} \mathcal{D}_{D}[h \phi] .
$$

Thus the second identity shows that

$$
\mathcal{K}_{D}^{(1)}[\phi]=-\frac{\partial}{\partial T}\left(h \frac{\partial \mathcal{S}_{D}[\phi]}{\partial T}\right)+\frac{\partial \mathcal{D}_{D}[h \phi]}{\partial \nu}+h \tau \mathcal{K}_{D}^{*}[\phi]-\mathcal{K}_{D}^{*}[h \tau \phi]
$$

for all $\phi \in H^{1}(\partial D)$. It is interesting to observe that the above identity tells us that the operator

$$
\phi \mapsto-\frac{\partial}{\partial T}\left(h \frac{\partial}{\partial T}\right) \mathcal{S}_{D}[\phi]+\frac{\partial}{\partial \nu} \mathcal{D}_{D}[h \phi]
$$

may be extended as a bounded operator on $L^{2}(\partial D)$.

\section{Asymptotic expansions of the GPTs}

We now derive asymptotic expansions of the GPTs.

Proposition 4.1. For multi-indices $\alpha$ and $\beta$, let $F(x)=x^{\beta}$ and $H(x)=x^{\alpha}$. Let

$$
\begin{aligned}
& \phi=\left(\lambda I-\mathcal{K}_{D}^{*}\right)^{-1}\left[\left.\frac{\partial H}{\partial \nu}\right|_{\partial D}\right], \\
& \psi=\left(\lambda I-\mathcal{K}_{D}\right)^{-1}\left[\left.F\right|_{\partial D}\right] .
\end{aligned}
$$

The following asymptotic expansion holds:

$$
M_{\alpha \beta}\left(k, D_{\epsilon}\right)-M_{\alpha \beta}(k, D)=\epsilon\left\langle h, p_{\alpha \beta}(k, D)\right\rangle_{L^{2}(\partial D)}+O\left(\epsilon^{2}\right),
$$

where

$$
\begin{aligned}
p_{\alpha \beta}(k, D)=\frac{\partial \psi}{\partial T} & \frac{\partial\left(H+\mathcal{S}_{D}[\phi]\right)}{\partial T}+\phi \frac{\partial\left(F+\mathcal{D}_{D}[\psi]\right)}{\partial \nu} \\
& +\psi\left(\left\langle\left(\nabla^{2} H\right) \nu, \nu\right\rangle+\left\langle\left(\nabla^{2} H\right) T, T\right\rangle\right) .
\end{aligned}
$$


Proof. Since

$$
F(x+\epsilon h(x) \nu(x))=F(x)+\epsilon h(x) \frac{\partial F}{\partial \nu}(x)+O\left(\epsilon^{2}\right), \quad x \in \partial D,
$$

it follows from (3.6) and Lemma 3.2 that

$$
\begin{aligned}
M_{\alpha \beta}\left(k, D_{\epsilon}\right)=\int_{\partial D_{\epsilon}} F(\tilde{x}) \tilde{\phi}_{\epsilon}(\tilde{x}) d \sigma_{\epsilon}(\tilde{x}) \\
\quad=\int_{\partial D}\left(F(x)+\epsilon h(x) \frac{\partial F}{\partial \nu}(x)\right)\left(\phi(x)+\epsilon \phi_{1}(x)\right)(1-\epsilon \tau(x) h(x)) d \sigma(x)+O\left(\epsilon^{2}\right) \\
\quad=M_{\alpha \beta}(k, D)+\epsilon \int_{\partial D} F \phi_{1} d \sigma+\epsilon \int_{\partial D}\left(\frac{\partial F}{\partial \nu}-\tau F\right) \phi h d \sigma+O\left(\epsilon^{2}\right) .
\end{aligned}
$$

Hence the definition (4.2) yields

$$
\begin{array}{r}
M_{\alpha \beta}\left(k, D_{\epsilon}\right)=M_{\alpha \beta}(k, D)+\epsilon \int_{\partial D}\left(\lambda I-\mathcal{K}_{D}\right)[\psi] \phi_{1} d \sigma \\
+\epsilon \int_{\partial D}\left(\frac{\partial F}{\partial \nu}-\tau F\right) \phi h d \sigma+O\left(\epsilon^{2}\right) .
\end{array}
$$

Let us now calculate the term $\int_{\partial D}\left(\lambda I-\mathcal{K}_{D}\right)[\psi] \phi_{1} d \sigma$. From (3.7) we get

$$
\begin{aligned}
\int_{\partial D}\left(\lambda I-\mathcal{K}_{D}\right)[\psi] \phi_{1} d \sigma & =\int_{\partial D} \psi\left(\lambda I-\mathcal{K}_{D}^{*}\right)\left[\phi_{1}\right] d \sigma \\
& =\int_{\partial D} \psi\left[\mathcal{K}_{D}^{(1)}[\phi]+h\left\langle\left(\nabla^{2} H\right) \nu, \nu\right\rangle-h^{\prime} \frac{\partial H}{\partial T}\right] d \sigma
\end{aligned}
$$

Next, because of (3.8), we have

$$
\int_{\partial D} \psi \mathcal{K}_{D}^{(1)}[\phi] d \sigma=\int_{\partial D} \psi\left[-\frac{\partial}{\partial T}\left(h \frac{\partial \mathcal{S}_{D}[\phi]}{\partial T}\right)+\frac{\partial \mathcal{D}_{D}[h \phi]}{\partial \nu}+h \tau \mathcal{K}_{D}^{*}[\phi]-\mathcal{K}_{D}^{*}[h \tau \phi]\right] d \sigma .
$$

We claim that

$$
\int_{\partial D} \psi \frac{\partial \mathcal{D}_{D}[h \phi]}{\partial \nu} d \sigma=\int_{\partial D} \frac{\partial \mathcal{D}_{D}[\psi]}{\partial \nu} h \phi d \sigma .
$$

In fact, let $\Lambda_{D}$ denote the Dirichlet-to-Neuman map on $D$, that is, $\Lambda_{D}[\psi]=\partial u / \partial \nu$, where $\Delta u=0$ in $D$ and $u=\psi$ on $\partial D$. Then Green's theorem yields

$$
\begin{aligned}
\int_{\partial D} \psi \frac{\partial \mathcal{D}_{D}[h \phi]}{\partial \nu} d \sigma & =\left.\int_{\partial D} \Lambda_{D}[\psi] \mathcal{D}_{D}[h \phi]\right|_{-} d \sigma \\
& =\int_{\partial D} \Lambda_{D}[\psi]\left(\frac{1}{2} I+\mathcal{K}_{D}\right)[h \phi] d \sigma \\
& =\int_{\partial D}\left(\frac{1}{2} I+\mathcal{K}_{D}^{*}\right) \Lambda_{D}[\psi] h \phi d \sigma
\end{aligned}
$$

In view of (2.2), the solution to the Dirichlet problem $\Delta u=0$ in $D$ and $u=\psi$ on $\partial D$ is given by

$$
u(x)=\mathcal{D}_{D}\left(\frac{1}{2} I+\mathcal{K}_{D}\right)^{-1}[\psi](x), \quad x \in D .
$$

Therefore, we have

$$
\Lambda_{D}[\psi]=\frac{\partial}{\partial \nu} \mathcal{D}_{D}\left(\frac{1}{2} I+\mathcal{K}_{D}\right)^{-1}[\psi] \quad \text { on } \partial D .
$$


It then follows from (2.1) that

$$
\left(\frac{1}{2} I+\mathcal{K}_{D}^{*}\right) \Lambda_{D}[\psi]=\left.\frac{\partial}{\partial \nu} \mathcal{S}_{D}\left[\frac{\partial}{\partial \nu} \mathcal{D}_{D}\left(\frac{1}{2} I+\mathcal{K}_{D}\right)^{-1}[\psi]\right]\right|_{+}
$$

One can easily see, using again Green's theorem and (2.2), that for $x \in \mathbb{R}^{2} \backslash \bar{D}$,

$$
\mathcal{S}_{D}\left[\frac{\partial}{\partial \nu} \mathcal{D}_{D}\left(\frac{1}{2} I+\mathcal{K}_{D}\right)^{-1}[\psi]\right](x)=\mathcal{D}_{D}\left[\left.\mathcal{D}_{D}\left(\frac{1}{2} I+\mathcal{K}_{D}\right)^{-1}[\psi]\right|_{-}\right](x)=\mathcal{D}_{D}[\psi](x) .
$$

Thus we get

$$
\left(\frac{1}{2} I+\mathcal{K}_{D}^{*}\right) \Lambda_{D}[\psi]=\frac{\partial \mathcal{D}_{D}[\psi]}{\partial \nu}
$$

and hence (4.6) holds.

With this result in hand, we now obtain

$$
\int_{\partial D} \psi \mathcal{K}_{D}^{(1)}[\phi] d \sigma=\int_{\partial D} h\left[\frac{\partial \psi}{\partial T} \frac{\partial \mathcal{S}_{D}[\phi]}{\partial T}+\frac{\partial \mathcal{D}[\psi]}{\partial \nu} \phi+\tau \psi \mathcal{K}_{D}^{*}[\phi]-\tau \mathcal{K}_{D}[\psi] \phi\right] d \sigma
$$

and hence

$$
\begin{aligned}
\int_{\partial D}\left(\lambda I-\mathcal{K}_{D}\right)[\psi] \phi_{1} d \sigma=\int_{\partial D} h & {\left[\frac{\partial \psi}{\partial T} \frac{\partial \mathcal{S}_{D}[\phi]}{\partial T}+\frac{\partial \mathcal{D}[\psi]}{\partial \nu} \phi+\tau \psi \mathcal{K}_{D}^{*}[\phi]\right.} \\
& \left.-\tau \mathcal{K}_{D}[\psi] \phi+\psi\left\langle\left(\nabla^{2} H\right) \nu, \nu\right\rangle+\frac{\partial}{\partial T}\left(\psi \frac{\partial H}{\partial T}\right)\right] d \sigma .
\end{aligned}
$$

It then follows from (4.5) that

$$
M_{\alpha \beta}\left(k, D_{\epsilon}\right)-M_{\alpha \beta}(k, D)=\epsilon \int_{\partial D} h(x) p_{\alpha \beta}(k, D)(x) d \sigma+O\left(\epsilon^{2}\right),
$$

where

$$
\begin{aligned}
p_{\alpha \beta}(k, D)= & \frac{\partial \psi}{\partial T} \frac{\partial \mathcal{S}_{D}[\phi]}{\partial T}+\frac{\partial \mathcal{D}[\psi]}{\partial \nu} \phi+\tau \psi \mathcal{K}_{D}^{*}[\phi]-\tau \mathcal{K}_{D}[\psi] \phi \\
& +\psi\left\langle\left(\nabla^{2} H\right) \nu, \nu\right\rangle+\frac{\partial}{\partial T}\left(\psi \frac{\partial H}{\partial T}\right)+\left(\frac{\partial F}{\partial \nu}-\tau F\right) \phi
\end{aligned}
$$

but,

$$
\frac{\partial}{\partial T}\left(\psi \frac{\partial H}{\partial T}\right)=\frac{\partial \psi}{\partial T} \frac{\partial H}{\partial T}+\psi\left\langle\left(\nabla^{2} H\right) T, T\right\rangle+\psi \tau \frac{\partial H}{\partial \nu} .
$$

Note also that because of (4.1) and (4.2),

$$
\mathcal{K}_{D}^{*}[\phi]+\frac{\partial H}{\partial \nu}=\lambda \phi \quad \text { and } \quad \mathcal{K}_{D}[\psi]+F=\lambda \psi
$$

Thus we arrive at

$$
\begin{aligned}
p_{\alpha \beta}(k, D)= & \frac{\partial \psi}{\partial T} \frac{\partial\left(H+\mathcal{S}_{D}[\phi]\right)}{\partial T}+\phi \frac{\partial\left(F+\mathcal{D}_{D}[\psi]\right)}{\partial \nu} \\
& +\psi\left(\left\langle\left(\nabla^{2} H\right) \nu, \nu\right\rangle+\left\langle\left(\nabla^{2} H\right) T, T\right\rangle\right),
\end{aligned}
$$

as desired. This completes the proof. 
Let us now suppose that $a_{\alpha}$ and $b_{\beta}$ are constants such that $H=\sum_{\alpha} a_{\alpha} x^{\alpha}$ and $F=\sum_{\beta} b_{\beta} x^{\beta}$ are harmonic polynomials. Then it can be easily seen that

$$
\begin{aligned}
\sum_{\alpha, \beta} a_{\alpha} b_{\beta} M_{\alpha \beta}\left(k, D_{\epsilon}\right)-\sum_{\alpha, \beta} a_{\alpha} b_{\beta} M_{\alpha \beta}(k, D) \\
=\epsilon\left\langle h, \sum_{\alpha, \beta} a_{\alpha} b_{\beta} p_{\alpha \beta}(k, D)\right\rangle_{L^{2}(\partial D)}+O\left(\epsilon^{2}\right),
\end{aligned}
$$

and

$$
\begin{aligned}
& \sum_{\alpha, \beta} a_{\alpha} b_{\beta} p_{\alpha \beta}(k, D) \\
& =\frac{\partial \psi}{\partial T} \frac{\partial\left(H+\mathcal{S}_{D}[\phi]\right)}{\partial T}+\phi \frac{\partial\left(F+\mathcal{D}_{D}[\psi]\right)}{\partial \nu}+\psi\left(\left\langle\left(\nabla^{2} H\right) \nu, \nu\right\rangle+\left\langle\left(\nabla^{2} H\right) T, T\right\rangle\right),
\end{aligned}
$$

where $\phi$ and $\psi$ satisfy (4.1) and (4.2) with new (harmonic functions) $H$ and $F$. Since $H$ is harmonic,

$$
\left\langle\left(\nabla^{2} H\right) \nu, \nu\right\rangle+\left\langle\left(\nabla^{2} H\right) T, T\right\rangle=\Delta H=0,
$$

and hence

$$
\sum a_{\alpha} b_{\beta} p_{\alpha \beta}(k, D)=\frac{\partial \psi}{\partial T} \frac{\partial\left(H+\mathcal{S}_{D}[\phi]\right)}{\partial T}+\phi \frac{\partial\left(F+\mathcal{D}_{D}[\psi]\right)}{\partial \nu} .
$$

Let

$$
u(x):=H(x)+\mathcal{S}_{D}[\phi](x) \text { and } \quad v(x):=F(x)+\mathcal{D}_{D}[\psi](x), \quad x \in \mathbb{R}^{2} .
$$

Then one can see using the jump relations (2.1) and (2.2) that $u$ and $v$ are, respectively, solutions to the following transmission problems:

$$
\begin{cases}\Delta u=0, & \text { in } D \cup\left(\mathbb{R}^{2} \backslash \bar{D}\right), \\ \left.u\right|_{+}-\left.u\right|_{-}=0, & \text { on } \partial D, \\ \left.\frac{\partial u}{\partial \nu}\right|_{+}-\left.k \frac{\partial u}{\partial \nu}\right|_{-}=0, & \text { on } \partial D \\ (u-H)(x)=O\left(|x|^{-1}\right) & \text { as }|x| \rightarrow \infty\end{cases}
$$

and

$$
\begin{cases}\Delta v=0, & \text { in } D \cup\left(\mathbb{R}^{2} \backslash \bar{D}\right), \\ \left.k v\right|_{+}-\left.v\right|_{-}=0, & \text { on } \partial D, \\ \left.\frac{\partial v}{\partial \nu}\right|_{+}-\left.\frac{\partial v}{\partial \nu}\right|_{-}=0, & \text { on } \partial D \\ (v-F)(x)=O\left(|x|^{-1}\right) & \text { as }|x| \rightarrow \infty\end{cases}
$$

From (4.10), (2.1) and (2.2) (see also [23]), we have

$$
\phi=\left.(k-1) \frac{\partial u}{\partial \nu}\right|_{-} \text {on } \partial D
$$

and

Hence it follows that

$$
\psi=\left.\frac{k-1}{k} v\right|_{-} \quad \text { on } \partial D .
$$

$$
\frac{\partial \psi}{\partial T}=\left.\frac{k-1}{k} \frac{\partial v}{\partial T}\right|_{-}
$$


In fact, from (4.2) we obtain that

$$
\left.v\right|_{-}=F+\left(\frac{1}{2} I+\mathcal{K}_{D}\right)[\psi]=\left(\lambda+\frac{1}{2}\right) \psi=\frac{k}{k-1} \psi .
$$

So far we proved the following theorem which is the main theoretical result of this paper.

Theorem 4.2. Suppose that $a_{\alpha}$ and $b_{\beta}$ are constants such that $H=\sum_{\alpha} a_{\alpha} x^{\alpha}$ and $F=\sum_{\beta} b_{\beta} x^{\beta}$ are harmonic polynomials. Then

$$
\begin{aligned}
& \sum_{\alpha, \beta} a_{\alpha} b_{\beta} M_{\alpha \beta}\left(k, D_{\epsilon}\right)-\sum_{\alpha, \beta} a_{\alpha} b_{\beta} M_{\alpha \beta}(k, D) \\
& =\epsilon(k-1) \int_{\partial D} h(x)\left[\left.\left.\frac{\partial v}{\partial \nu}\right|_{-} \frac{\partial u}{\partial \nu}\right|_{-}+\left.\left.\frac{1}{k} \frac{\partial u}{\partial T}\right|_{-} \frac{\partial v}{\partial T}\right|_{-}\right](x) d \sigma(x)+O\left(\epsilon^{2}\right),
\end{aligned}
$$

where $u$ and $v$ satisfy (4.11) and (4.12), respectively.

A few remarks are in order regarding the dependency of the remainder $O\left(\epsilon^{2}\right)$ term. It is bounded by $C \epsilon^{2}$ for some $C$ depending only on the $\mathcal{C}^{2}$-norm of $\partial D$ and $\|h\|_{\mathcal{C}^{1}(\partial D)}$. It also depends on the degrees of the harmonic polynomials $H$ and $F$. As the degree gets larger, the remainder gets larger. However, the remainder does not depend on the conductivity contrast $k$. Then formula (4.13) holds also for the extreme cases $k=0$ and $k=+\infty$. This important fact is because of the estimate

$$
\left\|\left(\frac{k+1}{2(k-1)} I-\mathcal{K}_{D}^{*}\right)^{-1}[f]\right\|_{L^{2}(\partial D)} \leq C\|f\|_{L^{2}(\partial D)}
$$

with a constant $C$ independent of $k$, which was first proved in [6].

Note also that formula (4.13) gives the shape derivative of $\sum_{\alpha, \beta} a_{\alpha} b_{\beta} M_{\alpha \beta}(k, D)$.

Finally, it is quite interesting to observe the similarity between the asymptotic formula (4.13) and the one for eigenvalue perturbations obtained in [1] (see [2] for the elasticity case).

\section{Reconstruction of Shape Details using GPTs}

5.1. Equivalent ellipse. As it is explained in [5], the polarization tensor $M$ can be explicitly computed for ellipses as follows. If $\mathcal{E}^{\prime}$ is an ellipse whose focal line is on the $x_{1}$-axis, its semi-major axis is of length $a$, and its semi-minor axis is of length $b$, then the polarization tensor of $\mathcal{E}^{\prime}$ is $M^{\prime}=(k-1)\left|\mathcal{E}^{\prime}\right|\left(\begin{array}{cc}\frac{a+b}{a+k b} & 0 \\ 0 & \frac{a+b}{b+k a}\end{array}\right)$. Now, let $\mathcal{E}=R \mathcal{E}^{\prime}$ where $R=\left(\begin{array}{cc}\cos \theta & -\sin \theta \\ \sin \theta & \cos \theta\end{array}\right)$ and $\theta \in[0, \pi]$, then the polarization tensor $M$ of $\mathcal{E}$ is $M=R M^{\prime} R^{T}$. Conversely, let $M$ be a positive definite symmetric matrix given, then the corresponding ellipse can be computed. Suppose that the eigenvalues of $M$ are $\lambda_{1}$ and $\lambda_{2}$ with $\lambda_{1}>\lambda_{2}$, and the corresponding eigenvectors of unit length are $\left(e_{11}, e_{12}\right)^{T}$ and $\left(e_{21}, e_{22}\right)^{T}$. Then $a=\sqrt{\frac{p}{\pi q}}, b=\sqrt{\frac{p q}{\pi}}, \theta=\arctan \frac{e_{21}}{e_{11}}$, where $\frac{1}{p}=\frac{k-1}{k+1}\left(\frac{1}{\lambda_{1}}+\frac{1}{\lambda_{2}}\right)$ and $q=\frac{\lambda_{2}-k \lambda_{1}}{\lambda_{1}-k \lambda_{2}}$.

Given the PT of the inclusion $B$, we can find an ellipse with the same PT but not its location since the PT is invariant under translation.

We can (approximately) locate the inclusion provided that its GPTs with $|\alpha|+$ $|\beta|=3$ are known. Suppose that $B=B_{r}\left(x^{*}\right)$ is a ball in $\mathbb{R}^{d}, d=2,3$. Let $i_{l}:=e_{l}$ and $j_{l}:=2 e_{l}, j=1, \ldots, d$. Then it is known (see [5]) that $\left(M_{i_{1} j_{1}}, \ldots, M_{i_{d} j_{d}}\right)=$ $\frac{2 d(k-1)}{k+d-1}|B| x^{*}$. Now, to locate the initial guess for an arbitrary-shaped target $B$, we 
consider as a first approximation that it is a ball and get the center for its initial guess from the measured $M_{i_{l} j_{l}}, l=1, \ldots, d$.

5.2. Recursive scheme. According to Theorem 2.1, we can (approximately) reconstruct the shape of $B$ by recursively minimizing at each step $n$ the functional $J^{(n)}[D]$ given in (1.4) over $D$. For fixed $n$, we make only one iteration to minimize $J^{(n)}[D]$. We modify the initial shape $D^{\text {init }}$ to obtain $D^{\text {mod }}$ by applying the gradient descent method. For doing so, we use

$$
\partial D^{\mathrm{mod}}=\partial D^{\mathrm{init}}-\left(\frac{J^{(n)}\left[D^{(\mathrm{init})}\right]}{\sum_{j}\left(\left\langle d_{S} J^{(n)}\left[D^{\text {init }}\right], \psi_{j}\right\rangle\right)^{2}} \sum_{j}\left\langle d_{S} J^{(n)}\left[D^{\text {init }}\right], \psi_{j}\right\rangle \psi_{j}\right) \nu
$$

where $\nu$ is the outward unit normal to $D^{\text {init }}$ and $\left\{\psi_{j}\right\}$ is a basis of $L^{2}\left(\partial D^{\text {init }}\right)$. For doing so, we need to compute the shape derivative of $J^{(n)}[D]$.

Let $H=\sum a_{\alpha} x^{\alpha}$ and $F=\sum b_{\beta} x^{\beta}$ be homogeneous harmonic polynomials and let

$$
\phi_{H F}(x)=(k-1)\left[\left.\left.\frac{\partial v}{\partial \nu}\right|_{-} \frac{\partial u}{\partial \nu}\right|_{-}+\left.\left.\frac{1}{k} \frac{\partial u}{\partial T}\right|_{-} \frac{\partial v}{\partial T}\right|_{-}\right],
$$

where $u$ and $v$ satisfy (4.11) and (4.12), respectively.

Theorem 4.2 shows that the shape derivative of $J^{(n)}[D]$ is given by

$$
\left\langle d_{S} J^{(n)}[D], h\right\rangle_{L^{2}(\partial D)}=\sum_{|\alpha|+|\beta| \leq K} w_{|\alpha|+|\beta|}^{(n)} \delta_{H F}\left\langle\phi_{H F}, h\right\rangle_{L^{2}(\partial D)},
$$

where

$$
\delta_{H F}=\sum_{\alpha, \beta} a_{\alpha} b_{\beta} M_{\alpha \beta}(k, D)-\sum_{\alpha, \beta} a_{\alpha} b_{\beta} M_{\alpha \beta}(k, B) .
$$

We now apply (5.1) where we replace the shape derivative by its expression in (5.2). Note that in $\delta_{H F}$, the GPTs of $B$ are from the measurements and those associated to $D$ are obtained from solving the boundary integral equation (2.3). Since the only information about $h$ is the inner product with $\phi_{H F}$, components of $h$ orthogonal to $\phi_{H F}$ are not detectable; see Figures 1 and 2.

Therefore, we form the (truncated) basis set $\left\{\psi_{j}\right\}$ as the collection of $\phi_{H F}$ with nonzero $w_{|\alpha|+|\beta|}^{(n)}$.

The equivalent ellipse obtained as in Section 5.1 can be used as an (initial) guess. More shape details for $B$ can be reconstructed by minimizing (1.4) by increasing $n$. The result of step $n-1$ is used as an initial guess for step $n$.

The weights $w_{|\alpha|+|\beta|}^{(n)}$ determine the GPTs we keep at each step. We choose in step $K-2 \geq n \geq 3$,

$$
w_{|\alpha|+|\beta|}^{(n)}=1 \quad \text { for } 2 \leq|\alpha|+|\beta| \leq n+2 \text { and } 0 \text { elsewhere. }
$$

If there are multiple inclusions, we choose in the second step

$$
w_{|\alpha|+|\beta|}^{(2)}=1 \quad \text { for } 3 \leq|\alpha|+|\beta| \leq K
$$

in order to have a better initial guess than an ellipse and, in step $K \geq n \geq 3$,

$$
w_{|\alpha|+|\beta|}^{(n)}=1 \quad \text { for } 2 \leq|\alpha|+|\beta| \leq n .
$$

Our algorithm is in the same spirit as the continuation method in frequency for solving inverse scattering problems [14, 15, 11. Since the high-frequency oscillations 
of the boundary of an inclusion are only contained in its high-order GPTs, our recursive optimization scheme yields a stable way to reconstruct such information.

5.3. Deformations undetectable from the GPTs. It follows from the expression of the shape derivative of $J^{(n)}$ that if a shape deformation is orthogonal to the functions $\phi_{H F}$, then it is undetectable to first order approximation.

As we can see from [9], if $D$ is a disk, then using $M_{\alpha \beta},|\alpha|+|\beta| \leq K$, we can only detect the Fourier coefficients of the deformation up to $K$.

Figures 1 and 2 are the (orthogonalized) $\phi_{H F}$ for $K=3$.
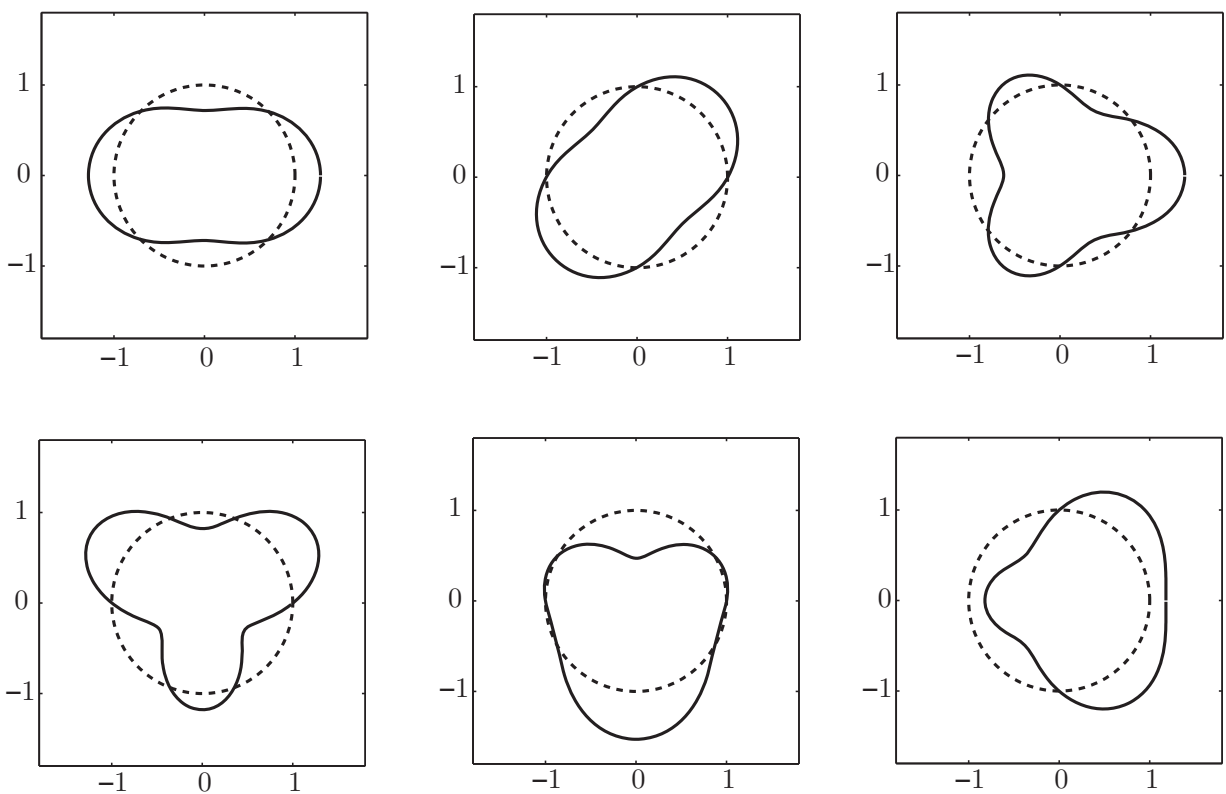

Figure 1. The basis of functions $\phi_{H F}$ for the search space used for the reconstructions from $\sum a_{\alpha} b_{\beta} M_{\alpha \beta}$, where $|\alpha|+|\beta| \leq$ 3 and $D$ is the dotted disk. The solid curves are $\partial D+$ a linear combination of $\phi_{H F} \nu$ and represent all the detectable shape variations.

\section{Numerical RESUlts}

In this section, we illustrate our algorithm for recovering the shape of a domain from its GPTs. In all of the numerical examples presented in this section, we apply the reconstruction scheme of multiple inclusions without making any a priori assumption on the number of connected components of the target. Moreover, in each step, we update the shape only one time using (5.1). More iterations do not yield a significantly better result.

In order to acquire the GPTs, we solve the boundary integral equation (2.3) numerically. After discretizing the boundary of a given simply connected domain $D$, the kernel functions involved in $\mathcal{K}_{D}^{*}$ and $\nu_{x} \cdot \nabla x^{\alpha}$ are evaluated at each nodal point on $\partial D$. If we call the obtained matrix and vector as $K^{d}$ and $B^{d}$, respectively, 

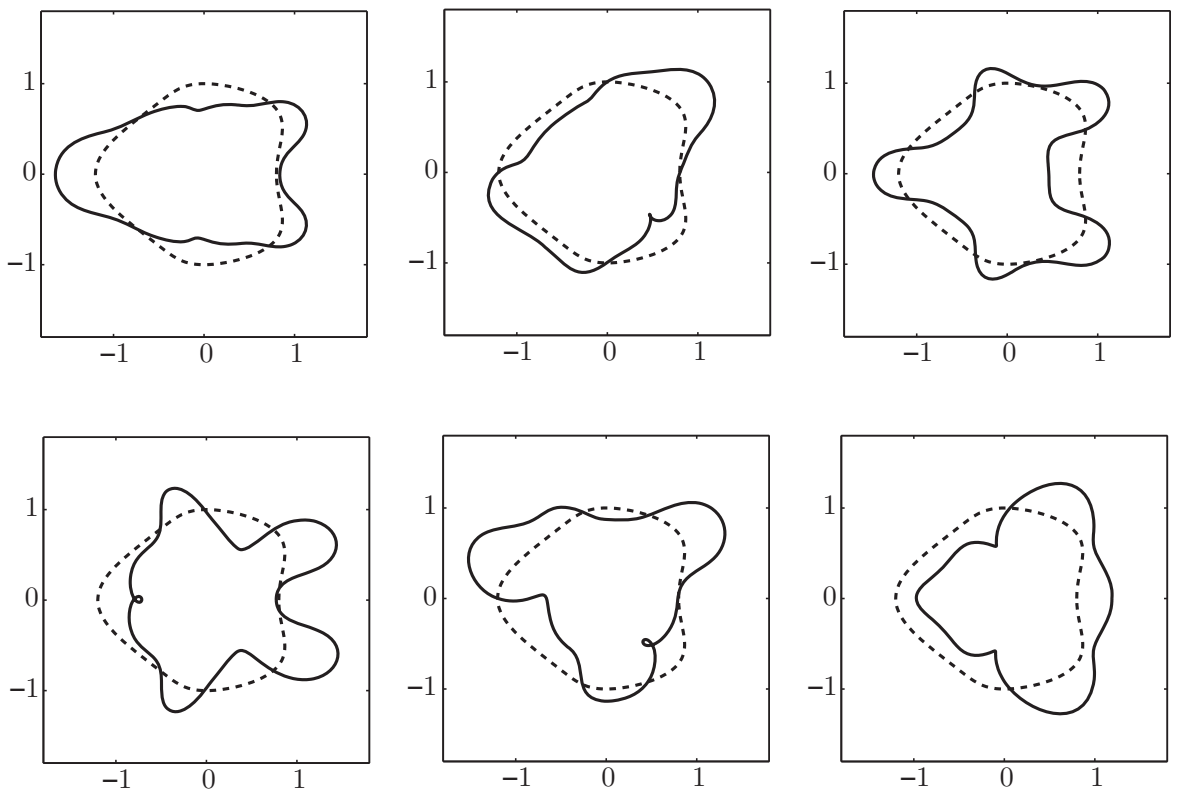

Figure 2. The basis of functions $\phi_{H F}$ for the search space used for the reconstructions from $\sum a_{\alpha} b_{\beta} M_{\alpha \beta}$, where $|\alpha|+|\beta| \leq$ 3 and $D$ is the dotted curve. The solid curves are $\partial D+$ a linear combination of $\phi_{H F} \nu$ and represent all the detectable shape variations.

then we obtain the discretized $\phi_{\alpha}$ by solving the linear system $\left(\lambda I-K^{d}\right) \phi_{\alpha}^{d}=B^{d}$. Finally, the numerical integration of (2.4) yields the GPTs. For the case of multiple inclusions, we use the system of integral equations derived in [8].

It is worth noticing that in the numerical examples, we only consider smooth curves. If $X(t):[a, b] \rightarrow \mathbb{R}^{2}$ is the arclength parametrization of $\partial D$ and $X$ is $\mathcal{C}^{2}$-function, then

$$
X\left(t_{0}+h\right)=x+h T(x)+\frac{h^{2}}{2} \tau(x) \nu(x)+o\left(h^{2}\right), \quad x=X\left(t_{0}\right),
$$

where $T$ is the tangential vector, $\nu$ the normal vector, and $\tau$ the curvature. Here, $o\left(h^{2}\right)$ depends on $\|X\|_{\mathcal{C}^{2}}$. Therefore,

$$
\frac{\langle x-y, \nu(x)\rangle}{|x-y|^{2}}=-\frac{1}{2} \tau(x)+o(1) \quad \text { for } x=X\left(t_{0}\right) \text { and } y=X\left(t_{0}+h\right),
$$

and hence we use $-\frac{1}{4 \pi} \tau(x)$ at the nodal points for the diagonal of the matrix $K^{d}$.

In the following examples, we use the GPTs up to $|\alpha|+|\beta| \leq 6$, i.e., $K=6$. The coefficients $a_{\alpha}$ and $b_{\beta}$ are chosen to be such that $\sum_{\alpha} a_{\alpha} x^{\alpha}$ and $\sum_{\beta} b_{\beta} x^{\beta}$ are harmonic polynomials and hence coincide with $\cos$ and sin functions on the unit circle. The conductivity $k$ inside the inclusion is set to be 3 . To reconstruct multiple inclusions as well as a single inclusion, we use in the second step $w_{|\alpha|+|\beta|}^{(2)}=1$ for $3 \leq$ $|\alpha|+|\beta| \leq 6$ and, in step $3 \leq n \leq 6, w_{|\alpha|+|\beta|}^{(n)}=1$ for $2 \leq|\alpha|+|\beta| \leq n$. The gray curve is the target domain $(B)$ and the black curve $(D)$ is the reconstructed one. 
In order to avoid inverse crime, we use different numbers of nodal points in the direct and inverse solvers; 500 points are used in the forward solver and 200 points are used in the inverse solver. Indeed, we do not share the same nodal points in the direct and forward solvers. Moreover, in the recursive algorithm, we strongly rely on the shape derivative obtained from the asymptotic analysis which is independent of the numerical computations.

Example 1. The example in Figure 3 shows that the equivalent ellipse is gradually modified toward the target domain. The first image is the equivalent ellipse and the others are the reconstructed images for $n=2, \ldots, 6$.
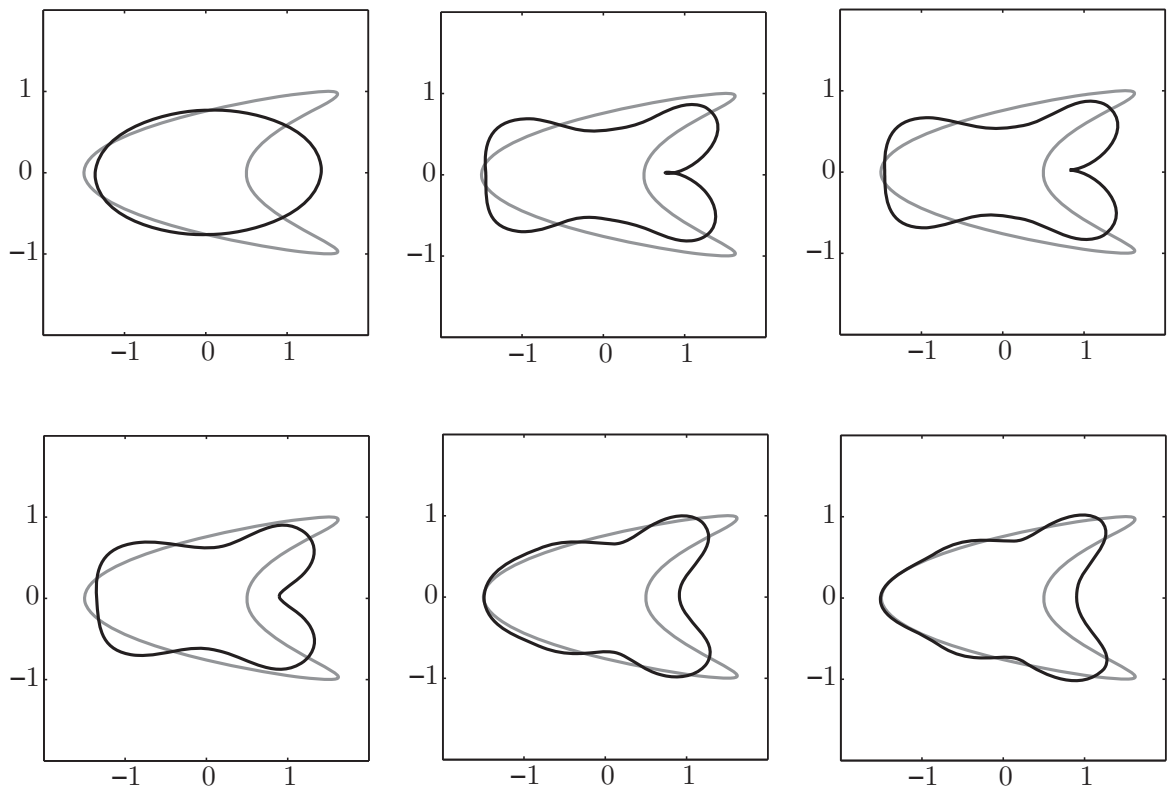

Figure 3. Gray curve is the target domain $(B)$ and the black curve $(D)$ is the reconstructed shape. The first image is the equivalent ellipse and the others are the reconstructed images for $n=2, \ldots, 6$. Here, the conductivity $k=3$.

Example 2. The example in Figure 4 shows the reconstruction of the kite-shaped inclusion with various relative errors $\frac{\left(\sum_{|\alpha|+|\beta| \leq 6}\left(\sum a_{\alpha} b_{\beta} \mathcal{E}_{\alpha \beta}\right)^{2}\right)^{\frac{1}{2}}}{\left(\sum_{|\alpha|+|\beta| \leq 6}\left(\sum a_{\alpha} b_{\beta} M_{\alpha \beta}^{\text {comp }}\right)^{2}\right)^{\frac{1}{2}}}=0,0.1,0.2$. Here $\mathcal{E}_{\alpha \beta}:=M_{\alpha \beta}^{\text {comp }}-M_{\alpha \beta}^{\text {data }}$, where $M_{\alpha \beta}^{\text {comp }}$ is the computed GPT and $M_{\alpha \beta}^{\text {data }}$ is the one used for the reconstruction. Figure 4 demonstrates the stability of the reconstruction procedure.

Example 3. The example in Figure 5 reveals the limitation of the shape reconstruction when we use the GPTs up to $|\alpha|+|\beta|=K$. When the target function is a sinusoidal perturbation of a disk, we can reconstruct the shape perturbation when the angular frequency is smaller than or equal to $K$. Higher-frequency information is undetectable. 

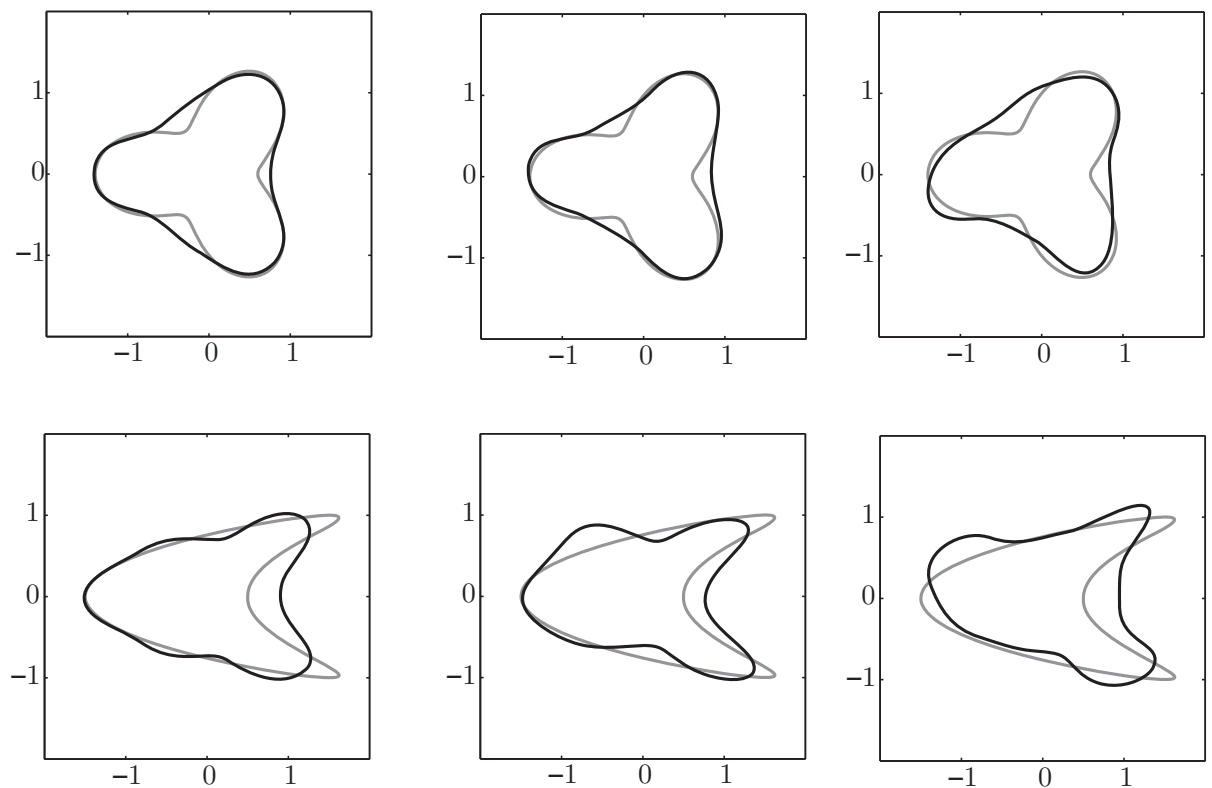

Figure 4. Gray curve is the target domain $(B)$ and the black curve $(D)$ is the reconstructed shape for $n=6$ starting from the equivalent ellipse. The first, second and third columns are from the data with $0 \%, 10 \%, 20 \%$ error, respectively. Here, the conductivity $k=3$.
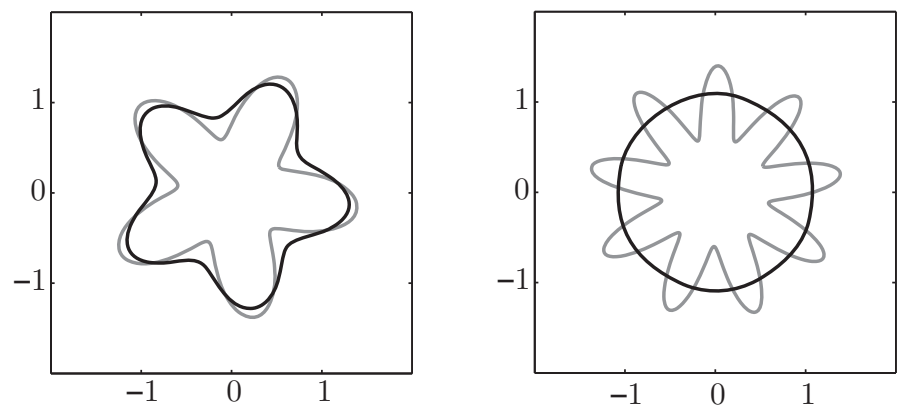

Figure 5. Gray curve is the target domain $(B)$ and the black curve $(D)$ is the reconstructed shape for $n=6$ starting from the equivalent ellipse. Here, the conductivity $k=3$.

Example 4. Figure 6 shows that it is more difficult to reconstruct high contrast inclusions. In order to obtain satisfactory reconstructions, one shall use more GPTs.

Example 5. Using higher-order GPTs we can better detect multiple inclusions; see Figure 7. Note that here, in order to generate the GPTs we need to modify 

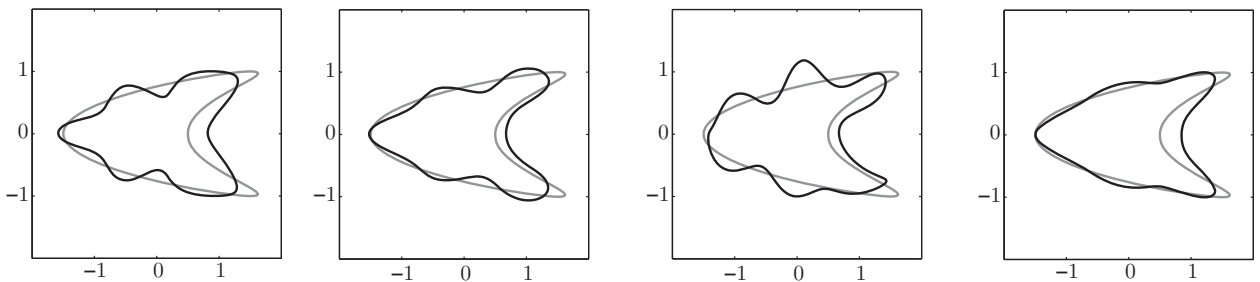

Figure 6. Reconstruction results for high contrast inclusions. Left to right: $(k=0.3, n=6),(k=0.3, n=9),(k=10, n=6)$, and $(k=10, n=9)$.
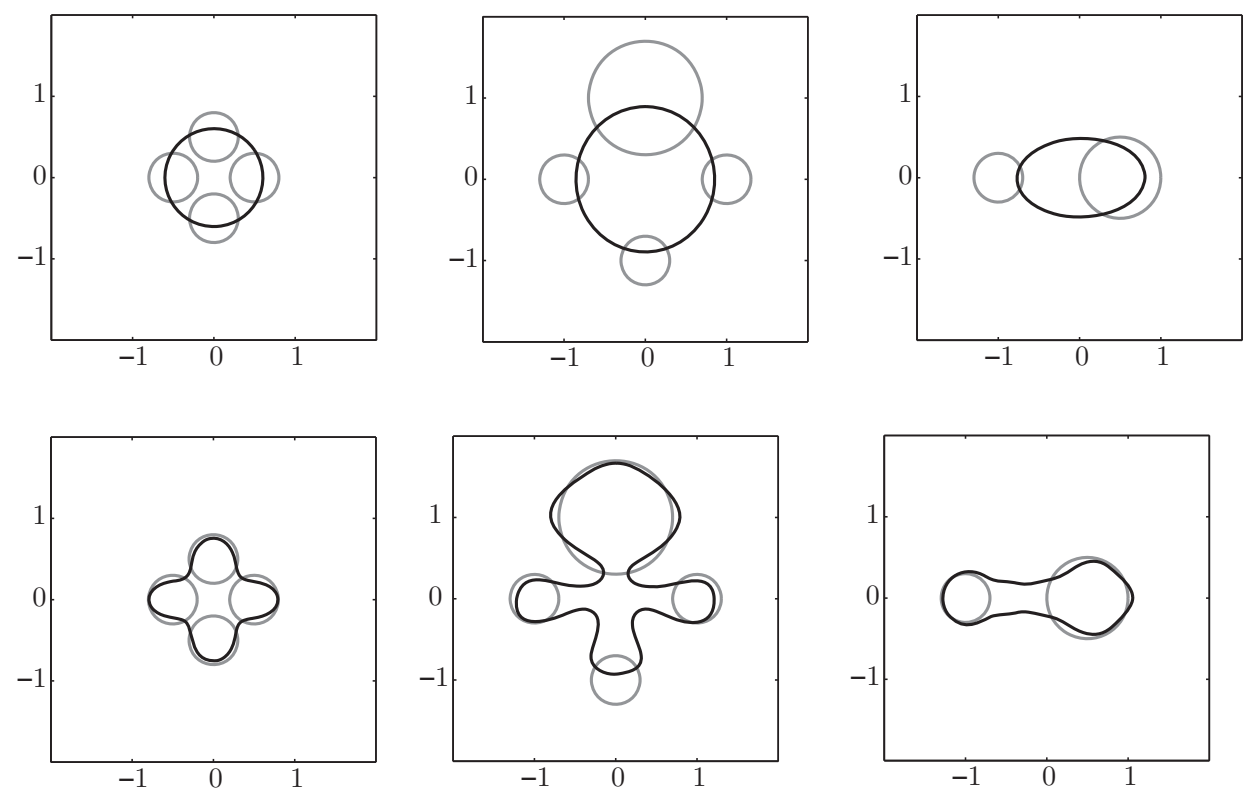

FiguRE 7. Reconstruction results for multiple inclusions with the same conductivity $k=3$. The upper images show the equivalent ellipse and the lower ones show the reconstructed image for $n=6$.

(2.3). In fact, we have to solve the following system of integral equations [8]:

$$
\left(\lambda_{j} I-\mathcal{K}_{D_{j}}^{*}\right)\left[\phi_{\alpha}^{(j)}\right](x)+\sum_{i \neq j} \frac{\partial \mathcal{S}_{D_{i}}\left[\phi_{\alpha}^{(i)}\right]}{\partial \nu^{(j)}}(x)=\nu_{x}^{(j)} \cdot \nabla x^{\alpha}, \quad x \in \partial D_{j}, \quad j=1, \ldots, m,
$$

where $m$ is the number of inclusions, $\nu^{(j)}$ the outward normal to $\partial D_{j}$, and $\lambda_{j}=$ $\left(k_{j}-1\right) /\left(2\left(k_{j}+1\right)\right)$ with $k_{j}$ being the conductivity of the inclusion $D_{j}$.

Example 6. Figure 8 shows a result for multiple inclusions with different conductivities. The conductivities are set to be $3,3,7,10$ in, respectively, $D_{1}, D_{2}, D_{3}, D_{4}$. As expected, the reconstructed image is pushed towards the inclusions with the highest conductivities. 

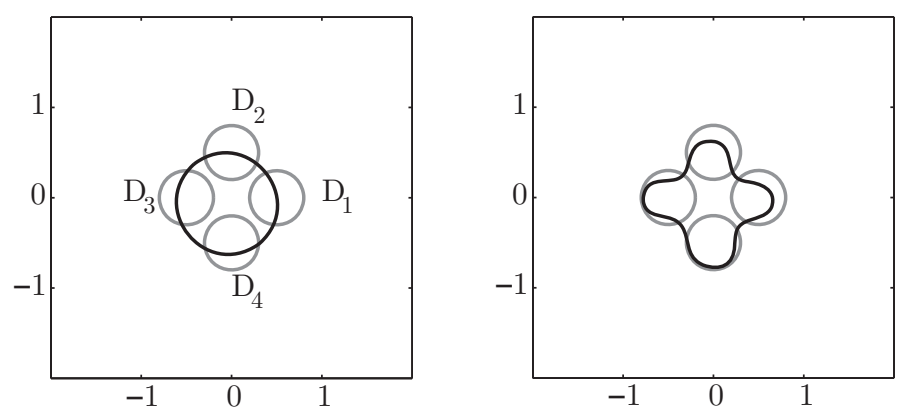

FiguRE 8. Reconstruction result for multiple inclusions with different conductivities. The conductivities are set to be $3,3,7,10$ in respectively $D_{1}, D_{2}, D_{3}, D_{4}$. The image on the left shows the equivalent ellipse and the one on the right shows the reconstructed image for $n=6$.

\section{Conclusion}

In this paper we have proposed a new recursive optimization scheme to recover fine shape details from the GPTs. We have presented results of some numerical experiments to demonstrate the validity and the limitations of the proposed approach which is in the same spirit as the continuation method in frequency. Since the high-frequency oscillations of the boundary of an inclusion are only contained in its high-order GPTs, the recursive method yields a stable way to reconstruct such information.

Other schemes can be designed by choosing different weights in the discrepancy functional (1.4). For example, choosing

$$
\begin{array}{ll}
w_{|\alpha|+|\beta|}^{(1)}=1 & \text { for } 2 \leq|\alpha|+|\beta| \leq l_{1} \text { and } 0 \text { elsewhere, } \\
w_{|\alpha|+|\beta|}^{(2)}=1 & \text { for } l_{1}+1 \leq|\alpha|+|\beta| \leq l_{2} \text { and } 0 \text { elsewhere, } \\
w_{|\alpha|+|\beta|}^{(3)}=1 & \text { for } l_{2}+1 \leq|\alpha|+|\beta| \leq l_{3} \text { and } 0 \text { elsewhere, }
\end{array}
$$

and so on, where $2<l_{1}<l_{2}<l_{3}<\ldots$, yields a scheme that is closely related to the one developed in [12. It could have better resolution than the one implemented in this paper but clearly is less stable. It requires a very good initial guess. A detailed resolution and stability analysis for both schemes will be reported elsewhere.

As will be shown in Part II [7, our approach in this paper can be very nicely extended to the reconstruction problem of the electromagnetic parameters and the shape of a target from multi-static response matrix measurements at a single frequency when the target is of characteristic size less than the operating wavelength. It would be very interesting to generalize our approach to elasticity imaging.

\section{ACKNowledgements}

The authors are very grateful to the reviewers for their comments and suggestions to improve the presentation of the paper. The work of HA and HK was supported in part by Korea Research Foundation through grant KRF-2008-220-C00002. This work was partly supported by the second stage of the Brain Korea 21 Project, the 
Development Project of Human Resources in Mathematics, KAIST in 2010, and the Korea Science and Engineering Foundation (KOSEF) grant funded by the Korea government (MEST) (No. 2009-0070442).

\section{REFERENCES}

[1] H. Ammari, E. Beretta, E. Francini, H. Kang, and M. Lim, Optimization algorithm for reconstructing interface changes of a conductivity inclusion from modal measurements, Math. Comp., 79 (2010), 1757-1777. MR2630011

[2] H. Ammari, E. Beretta, E. Francini, H. Kang, and M. Lim, Reconstruction of small interface changes of an inclusion from modal measurements II: The elastic case, J. Math. Pures Appl., 94 (2010), 322-339. MR2679030

[3] H. Ammari and H. Kang, High-order terms in the asymptotic expansions of the steady-state voltage potentials in the presence of conductivity inhomogeneities of small diameter, SIAM J. Math. Anal., 34 (2003), 1152-1166. MR2001663 (2004e:35027)

[4] H. Ammari and H. Kang, Properties of generalized polarization tensors, SIAM Multiscale Model. Simul., 1 (2003), 335-348. MR1990200 (2004g:78029)

[5] H. Ammari and H. Kang, Reconstruction of Small Inhomogeneities from Boundary Measurements, Lecture Notes in Mathematics, Vol. 1846, Springer-Verlag, Berlin, 2004. MR 2168949 (2006k:35295)

[6] H. Ammari and H. Kang, Polarization and Moment Tensors with Applications to Inverse Problems and Effective Medium Theory, Applied Mathematical Sciences, Vol. 162, SpringerVerlag, New York, 2007. MR2327884(2009f:35339)

[7] H. Ammari, H. Kang, E. Kim, and J.Y. Lee, The generalized polarization tensors for resolved imaging. Part II: Shape and electromagnetic parameters reconstruction of an electromagnetic inclusion from multistatic measurements, Math. Comp., to appear.

[8] H. Ammari, H. Kang, E. Kim, and M. Lim, Reconstruction of closely spaced small inclusions, SIAM J. Numer. Anal., 42 (2005), 2408-2428. MR2139399 (2005m:78011)

[9] H. Ammari, H. Kang, M. Lim, and H. Zribi, Conductivity interface problems. Part I: Small perturbations of an interface, Trans. Amer. Math. Soc., 362 (2010), 2435-2449. MR2584606

[10] H. Ammari, H. Kang, and K. Touibi, Boundary layer techniques for deriving the effective properties of composite materials, Asymp. Anal., 41 (2005), 119-140. MR 2129229 (2006b:35333)

[11] G. Bao, F. Ma, and Y. Chen, An error estimate for recursive linearization of the inverse scattering problems, J. Math. Anal. Appl., 247 (2000), 255-271.

[12] L. Borcea, G. Papanicolaou, and F.G. Vasquez, Edge illumination and imaging of extended reflectors, SIAM J. Imaging Sci., 1 (2008), 75-114. MR2475826(2010a:15028)

[13] M. Brühl, M. Hanke, and M.S. Vogelius, A direct impedance tomography algorithm for locating small inhomogeneities, Numer. Math., 93 (2003), 635-654. MR.1961882 (2004b:65169)

[14] Y. Chen and V. Rokhlin, On the inverse scattering problem for the Helmholtz equation in one dimension, Inverse Problems, 8 (1992), 365-391. MR1166487 (93c:34034)

[15] R.R. Coifman, M. Goldberg, T. Hrycak, M. Israel, and V. Rokhlin, An improved operator expansion algorithm for direct and inverse scattering computations, Waves Random Media, 9 (1999), 441-457. MR.1705850(2000f:65151)

[16] D. Colton and R. Kress, Integral Equation Methods in Scattering Theory, John Wiley \& Sons Inc, 1983. MR700400 (85d:35001)

[17] R. Coifman, A. McIntosh, and Y. Meyer, L'intégrale de Cauchy définit un opérateur borné sur $L^{2}$ pour les courbes Lipschitziennes, Ann. Math., 116 (1982), 361-387. MR672839 (84m:42027)

[18] E.B. Fabes, M. Jodeit, and N.M. Riviére, Potential techniques for boundary value problems on $\mathcal{C}^{1}$ domains, Acta Math., 141 (1978), 165-186. MR.501367 (80b:31006)

[19] A. Friedman and M.S. Vogelius, Identification of small inhomogeneities of extreme conductivity by boundary measurements: a theorem on continuous dependence, Arch. Rat. Mech. Anal., 105 (1989), 299-326. MR973245 (90c:35198)

[20] G.B. Folland, Introduction to Partial Differential Equations, Princeton University Press, Princeton, New Jersey, 1976. MR 0599578 (58:29031)

[21] V. Isakov, On uniqueness of recovery of a discontinuous conductivity coefficient, Comm. Pure Appl. Math., 41 (1988), 865-877. MR951742 (90f:35205) 
[22] G.W. Milton, The Theory of Composites, Cambridge Monographs on Applied and Computational Mathematics, Cambridge University Press, 2001. MR.1899805 (2003d:74077)

[23] H. Kang and J.K. Seo, Recent progress in the inverse conductivity problem with single measurement, in Inverse Problems and Related Fields, CRC Press, Boca Raton, FL, 69-80, 2000. MR1761339 (2001f:35427)

[24] M. Lim, K. Louati, and H. Zribi, Reconstructing small perturbations of scatterers from electric or acoustic far-field measurements. Math. Meth. Appl. Sci., 31 (2008), 1315-1332. MR2431429 (2009h:35449)

[25] G. Pólya and G. Szegö, Isoperimetric Inequalities in Mathematical Physics, Annals of Mathematical Studies Number 27, Princeton University Press, Princeton, NJ, 1951. MR 0043486 $(13: 270 \mathrm{~d})$

[26] G.C. Verchota, Layer potentials and boundary value problems for Laplace's equation in Lipschitz domains, J. Funct. Anal., 59 (1984), 572-611. MR769382 (86e:35038)

Department of Mathematics and Applications, Ecole Normale Supérieure, 45 Rue D'Ulm, 75005 Paris, France

E-mail address: habib.ammari@ens.fr

Department of Mathematics, Inha University, Incheon 402-751, Korea

E-mail address: hbkang@inha.ac.kr

Department of Mathematical Sciences, Korean Advanced Institute of Science and Technology, Daejeon 305-701, Korea

E-mail address: mklim@kaist.ac.kr

Department of Mathematical Sciences, Korean Advanced Institute of Science and Technology, Daejeon 305-701, Korea

E-mail address: zribi@cmapx.polytechnique.fr 